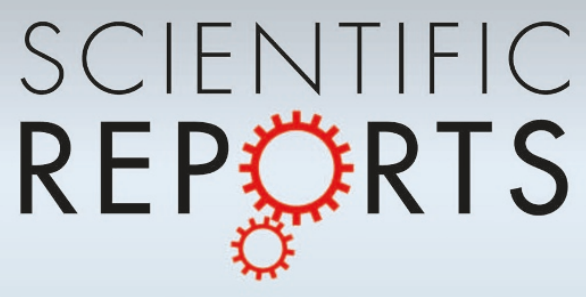

OPEN

SUBJECT AREAS:

METAMATERIALS

ELECTRICAL AND ELECTRONIC

ENGINEERING

TERAHERTZ OPTICS

Received

1 October 2014

Accepted

10 December 2014

Published

13 January 2015

Correspondence and requests for materials should be addressed to M.N.-C. (m.navarro@ imperial.ac.uk)

\title{
Planar Holographic Metasurfaces for Terahertz Focusing
}

\author{
Sergei A. Kuznetsov ${ }^{1,2,3}$, Mikhail A. Astafev ${ }^{1,2}$, Miguel Beruete ${ }^{4}$ \& Miguel Navarro-Cía ${ }^{5,6,7}$
}

\begin{abstract}
${ }^{1}$ Novosibirsk State University, Pirogova St. 2, Novosibirsk, 630090, Russian Federation, ${ }^{2}$ Budker Institute of Nuclear Physics SB RAS, Lavrentiev Ave. 11, Novosibirsk, 630090, Russian Federation, ${ }^{3}$ Institute of Semiconductor Physics SB RAS, Novosibirsk Branch "TDIAM", Lavrentiev Ave. 2/1, Novosibirsk, 630090, Russian Federation, ${ }^{4}$ Antennas Group - TERALAB, Universidad Pública de Navarra, 31006 , Pamplona, Spain, ${ }^{5}$ Optical and Semiconductor Devices Group, Department of Electrical and Electronic Engineering, Imperial College London, SW7 2BT, London, UK, ${ }^{6}$ Centre for Plasmonics and Metamaterials, Imperial College London, London SW7 2AZ, United Kingdom, ${ }^{7}$ Centre for Terahertz Science and Engineering, Imperial College London, London SW7 2AZ, United Kingdom.
\end{abstract}

Scientists and laymen alike have always been fascinated by the ability of lenses and mirrors to control light. Now, with the advent of metamaterials and their two-dimensional counterpart metasurfaces, such components can be miniaturized and designed with additional functionalities, holding promise for system integration. To demonstrate this potential, here ultrathin reflection metasurfaces (also called metamirrors) designed for focusing terahertz radiation into a single spot and four spaced spots are proposed and experimentally investigated at the frequency of $0.35 \mathrm{THz}$. Each metasurface is designed using a computer-generated spatial distribution of the reflection phase. The phase variation within $360 \mathrm{deg}$ is achieved via a topological morphing of the metasurface pattern from metallic patches to U-shaped and split-ring resonator elements, whose spectral response is derived from full-wave electromagnetic simulations. The proposed approach demonstrates a high-performance solution for creating low-cost and lightweight beam-shaping and beam-focusing devices for the terahertz band.

$\mathrm{T}$ he terahertz (THz) band of the electromagnetic spectrum is conveniently located between the microwaves and infrared regions allowing researchers to combine both optical and microwave techniques in $\mathrm{THz}$ instrumentation and to synthesize unique instrumental solutions ${ }^{1}$. Such a synthesis is proven to be very effective when planar metallized microstructures of subwavelength topology, commonly referred to as frequency selective surfaces or metasurfaces ${ }^{2}$, are used for manipulating amplitude, polarization and phase characteristics of microwaves ${ }^{3-5}$, quasi-optical $\mathrm{THz}^{6-8}$ and optical beams ${ }^{9-12}$ as well as acoustic waves ${ }^{13}$. In microwave and millimetre-wave engineering, control of the phase is essential in the technology of low-profile reflectarray antennas, whose conventional design implies exploiting a metasurface laying over a grounded-dielectric-slab with spatialdependent reflection phase ${ }^{4,14,15}$. This reflection-type approach holds promise for $\mathrm{THz}$ beam-shaping and beam-focusing techniques as it allows creating purely flat, thin and lightweight reflectors capable of properly manipulating the wavefront through the control of the metasurface unit cell geometry. Due to relative simplicity in photolithographic fabrication, such kind of reflectors serve as an attractive alternative to the conventional diffractive optical elements (DOEs) based on cost-consuming structures with profiled surfaces ${ }^{16-18}$. In addition, working in reflection mode eliminates the Fresnel reflection loss and reduces the terahertz-material interaction. This lowers the overall insertion loss of the device compared to transmission metasurfaces.

In this paper we investigate the feasibility for effective focusing of terahertz waves using metasurface-inspired flat holographic reflectarrays (HRAs) (see Fig. 1), whose design is accomplished by using a combined approach involving: i) a computer-generated holography (CGH) method ${ }^{19-21}$ to synthesize an appropriate distribution of the reflection phase $\varphi_{H R A}(x, y)$ over the holographic metasurface; ii) a full-wave-electromagnetic-analysis-based method to control the complex reflectance $\rho_{H R A}(x, y)$ of the HRA at any local point $(x, y)$ of its surface via proper morphing the metasurface unit cell geometry from metallic patches ${ }^{14,22}$ to U-shaped ${ }^{23}$ and split-ring resonator elements ${ }^{7,24,25}$ akin to surface wave inhomogeneous metalenses ${ }^{26}$. Given the anisotropy of the U-shaped and splitring resonators, numerical investigation of the TM- and TE-polarization is done. We conclude that the TEpolarization scheme leads to a better overall performance due to its negligible cross polarization. We report experimentally two different metasurfaces designed for the reflective focusing of an incident TE-polarized Gaussian beam into a single spot and four spaced spots respectively, which were optimized for the operation frequency of $0.35 \mathrm{THz}$ (free-space wavelength $\lambda_{0}=857 \mu \mathrm{m}$ ) chosen due to its positioning in the atmospheric- 


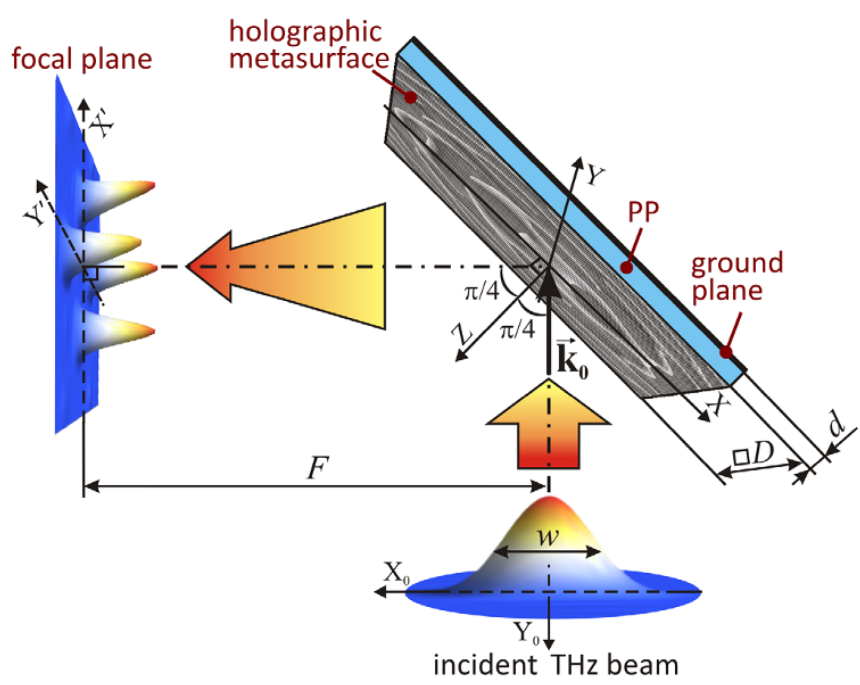

Figure $1 \mid 90$ deg-reflection schematic setup to focus a Gaussian terahertz beam by a metasurface. $\mathbf{k}_{\mathbf{0}}$ is the wave vector of the incident beam, having the following components in the $(\mathrm{X}, \mathrm{Y}, \mathrm{Z})$ coordinate system: $\mathbf{k}_{\mathbf{0}}=\left(-k_{0}\right)$ $\sqrt{2}, 0,-k_{0} / \sqrt{2}$ ), where $k_{0}=2 \pi / \lambda_{0}$. The plane (X, Y) is chosen to coincide with the outer interface of the metasurface.

transmission window ${ }^{27}$. An overall efficiency $\sim 80 \%$ is achieved in both cases, which outperforms that reported for transmission metasurfaces working at $\mathrm{THz}$ frequencies ${ }^{8}$.

\section{Design procedure and results}

To attain the desired intensity distribution $I_{F P}\left(x^{\prime}, y^{\prime}\right)$ in the focal plane $\left(\mathrm{X}^{\prime}, \mathrm{Y}^{\prime}\right)$ for the wavefront focused by the metasurface, the optimal surface distribution reflection phase $\varphi_{H R A}(x, y)$ referred to the $(\mathrm{X}, \mathrm{Y})$ plane can be effectively calculated by using CGH phase retrieval methods originally developed for profiled DOEs. In this work, we employed the easy-to-code and robust iterative algorithm of Gerchberg-Saxton (GSA) $)^{19-21}$, following the workflow shown in Fig. 2. This algorithm, although more complex than alternatives such as the standard ray-tracing method, is applicable for any configuration and, in particular, provides more accurate results in non-paraxial configurations like the one here reported.

To cover the range of $360 \mathrm{deg}$ variation of the reflection phase $\varphi_{H R A}$, which is necessary for accurate focusing, we continuously changed the metasurface unit cells geometry from square-shaped metallic patches ${ }^{14,22}$ to U-shaped resonators (USRs) ${ }^{23}$ and then to split-ring resonator elements $(\mathrm{SRRs})^{7,24,25}$, as it is shown in Fig. 3(a). As a dielectric substrate, a polypropylene (PP) slab of thickness $d=190 \mu \mathrm{m}\left(d \cong \lambda_{0} / 4.5\right)$ was employed to minimize dielectric losses $(\tan \Delta \approx 0.001)^{28,29}$. To minimize Ohmic dissipation, $0.35 \mu \mathrm{m}$ thick aluminum metallization was used both in the metasurface and ground plane layers. Due to a relatively low PP refractive index $n$ ( $\mathrm{n}$ $\cong 1.5$ ), such topological morphing ("Patch-to-SRR") enables to enhance subwalengthness of the unit cells compared to the patchbased RAs ${ }^{14,22}$ and, therefore, to decrease the phase errors when substituting the required local phase $\varphi_{H R A}(x, y)$ by the one retrieved from simulations of the regularly patterned surfaces. Moreover, when using only a single layer metasurface, it allows us to overcome the key drawback of the patch-based structures - impossibility to overlap $360 \mathrm{deg}$ for the reflection phase ${ }^{14,15}$. It should be also pointed out that the proposed metasurface based on "Patch-to-SRR" morphing is polarization-sensitive and supposed to be excited by the wave polarized transversely to USR and SRR gaps. Formally, a different morphology change involving for instance only isotropic unit cells could have been chosen at the expense of removing the polarizationdependence of the holographic metasurface. However, we found that "Patch-to-SRR" morphing enables to obtain smaller dimensions of the metasurface unit cells and, therefore, to enhance their subwalengthness, when the minimal feature size technologically allowable for the metasurface is fixed ( $56 \mu \mathrm{m}$ in this work).

Figure 3(b) illustrates variation of the absolute reflection phase $\varphi_{H R A}$ and the reflection coefficient $\left|\rho_{\text {HRA }}\right|^{2}$ induced by "Patch-toSRR" topological morphing of the optimized metasurface pattern at the frequency of $0.35 \mathrm{THz}$ simulated for the cases of TE- and TM-polarization. The morphing starts with a monotone increase of the patch width $p$ from 56 to $230 \mu \mathrm{m}$ (increment $\Delta p=6 \mu \mathrm{m}$ ). The largest patch then transforms to USR via forming a rectangular horizontal recess, varying $q$ from 6 to $174 \mu \mathrm{m}$ (increment $\Delta q=$ $6 \mu \mathrm{m})$. Finally, USR turns into SRR by extending the metallic arms of the USR gap, so that the width $r$ decreases from 110 to $56 \mu \mathrm{m}$ (increment $\Delta r=-3 \mu \mathrm{m}$ ). This three-step variation of the unit cell morphology is chosen to modify gradually the electromagnetic response such that the local periodicity approximation holds. For TEpolarization, such topological morphing leads to $360 \mathrm{deg}$ variation of the reflection phase from the initial patch to the final SRR, see Fig. 3(a). For TM-polarization, the phase excursion is $384 \mathrm{deg}$.
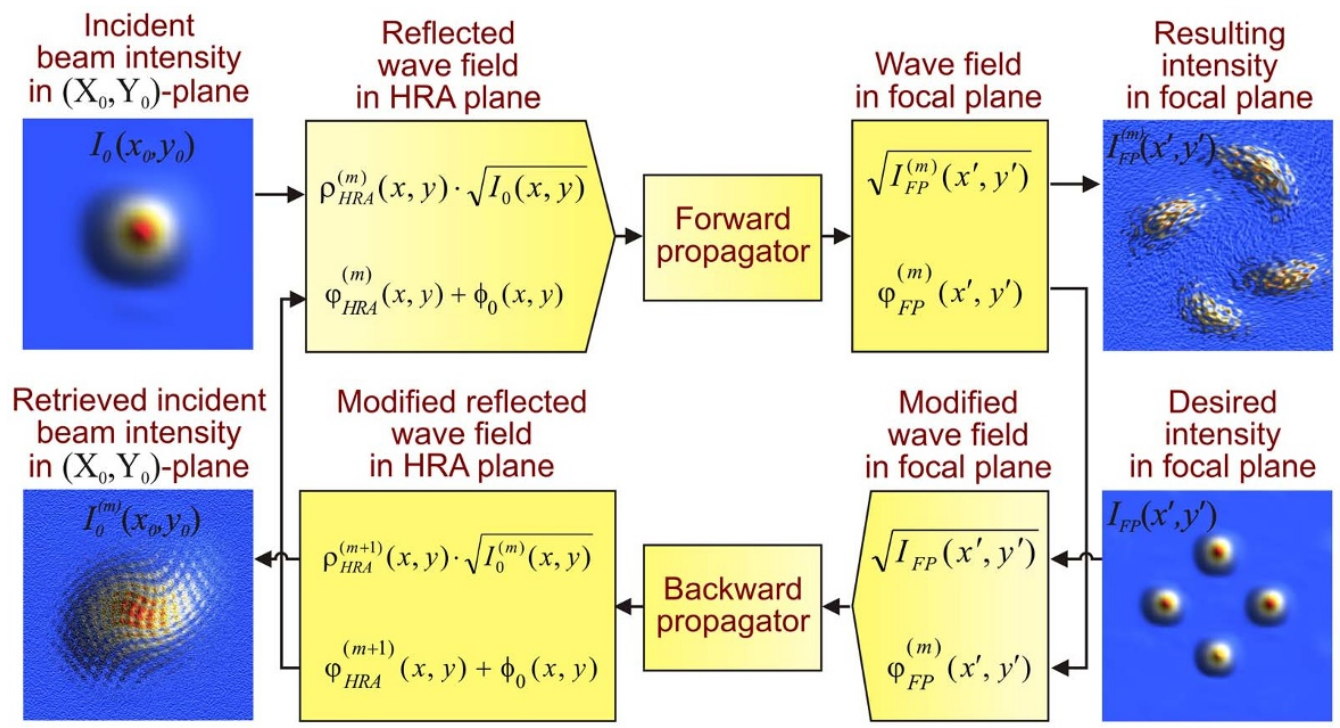

Figure $2 \mid$ The workflow of the Gerchberg-Saxton iterative algorithm for retrieving surface distribution of the HRA reflection phase $\varphi_{H R A}(x, y)$. 

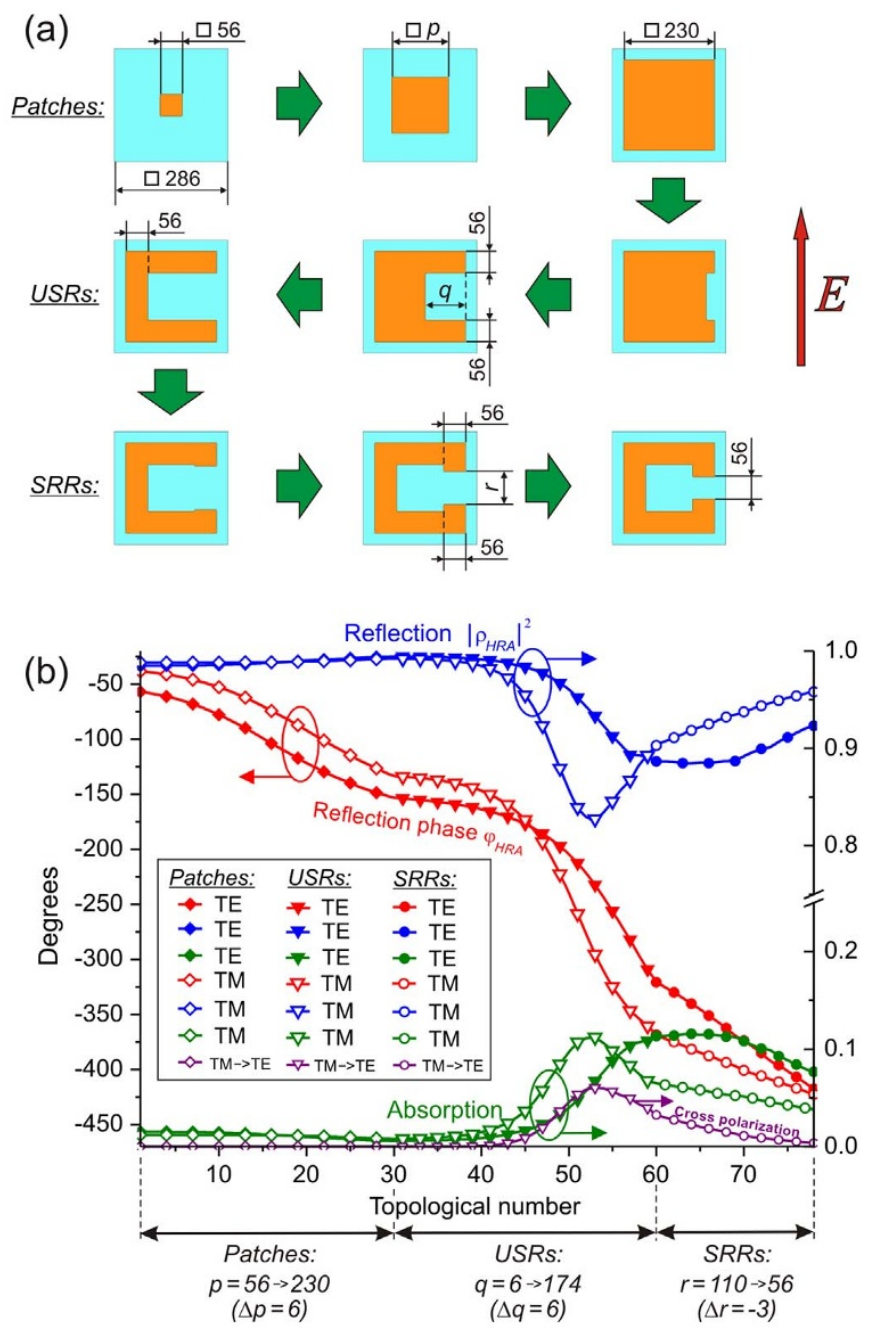

Figure 3 | "Patch-to-SRR" morphing. (a) "Patch-to-SRR" route for topological morphing of the metasurface pattern and (b) simulated performance of corresponding uniform-metasurface-based reflectarrays at the frequency of $0.35 \mathrm{THz}$. The exciting polarization is transverse to USR and SRR slots. The geometric parameters are given in micrometers. Metallization is shown in orange (dark).

A clear drawback of the TM case is the depolarization or crosspolarization (TM-to-TE conversion) under oblique incidence due to the intrinsic bianisotropy of the USR and SRR unit cells ${ }^{30}$. In Fig. 3(b) cross-polarization losses are clearly observed for USR and SRR cases, and are maximized for USR elements (6\% in the worst case). On the other hand, under TE excitation cross-polarization due to bianisotropy does not appear ${ }^{30}$ and thus the level of TE-to-TM conversion does not exceed $-60 \mathrm{~dB}\left(10^{-6}\right)$. Note that the cross-polarization losses decrease the TM-reflectivity down to $82.7 \%$ in contrast to $88.4 \%$ for the TE case. As for TE and TM absorption losses, whose values can be evaluated by the formulas $A_{T E}=1-\left|\rho_{H R A}\right|_{T E \rightarrow T E}^{2}-\left|\rho_{H R A}\right|_{T E \rightarrow T M}^{2}$ and $A_{T M}=1-\left|\rho_{H R A}\right|_{T M \rightarrow T M}^{2}-\left|\rho_{H R A}\right|_{T M \rightarrow T E}^{2}$ their maximal magnitudes reach $A_{T E}=11.5 \%$ and $A_{T M}=11.2 \%$ respectively (see Fig. 3(b)). The field analysis shows that absorption is attributed mainly to Ohmic dissipation in the metallization.

For the experimental confirmation of the proposed approach, the two kinds of metasurfaces designed were fabricated and experimentally investigated. In the first design an incident Gaussian beam is focused into a single spot (1-spot), whereas in the second it is divided into four spaced spots (4-spots) placed in the vertices of a symmetric cross with $12 \mathrm{~mm}$ axes. The metasurfaces were optimized for TEpolarized excitation to minimize cross-polarization losses.
Figure 4(a) displays the GSA-computed distributions of the reflection phase $\varphi_{H R A}(x, y)$ over the metasurfaces for a focal distance $F=60 \mathrm{~mm}$. When realizing GSA, which typically converged after 20 iterations, the metasurface was spatially discretized into uniform pixels with dimensions $286 \times 286 \mu \mathrm{m}$ equal to the dimensions of the unit cells. This discretization step was used in the FresnelKirchhoff surface integral computation and the phase was assumed to be constant within the pixel area. After computing the phase distribution $\varphi_{H R A}(x, y)$, a photomask was generated with a MATLAB $^{\mathrm{TM}}$ code by using the correspondence between the phase and the unit cell geometry represented in Fig. 3. This photomask was used for the fabrication of the metallic pattern via a photolithographic technique ${ }^{28,29}$. As only a relative phase shift between pixels makes sense, the phase of $180 \mathrm{deg}$ was chosen to refer to the $1 \mathrm{st}$ number topology ("patch", $p=56 \mu \mathrm{m}$ ), while the 78th topology ("SRR", $w=56 \mu \mathrm{m}$ ) corresponded to the phase of $-180 \mathrm{deg}$. Figure 4(b) shows a detail of the actual mask pattern corresponding to the 4-spots design.

To evaluate the focusing performance, an experimental setup implemented with CDP System Corp. components ${ }^{32}$ and utilizing a quasi-optical BWO-spectroscopy technique ${ }^{33}$ was employed (Fig. 5). In this setup, the measurements were carried out with a polarization-insensitive pyroelectric sensor mounted on the tip of a metallic rod with length of $50 \mathrm{~mm}$ and outer diameter of $15 \mathrm{~mm}$ installed on a computer-controlled 2D raster scanner. The pyrosensor was designed to have a receiving window diameter of $1.5 \mathrm{~mm}$. The polarization of the BWO beam incident upon the metasurface was controlled with a wire grid polarizer.

The theoretical and experimentally measured focusing characteristics for 1-spot and 4-spots designs are demonstrated in Fig. 6 where the intensity distributions $I_{F P}\left(x^{\prime}, y^{\prime}\right)$ in the focal plane $\left(\mathrm{X}^{\prime}, \mathrm{Y}^{\prime}\right)$ are shown. The distributions are normalized to the intensity of the incident TE-polarized Gaussian beam. By inspection of Fig. 6, we conclude that there is good agreement between simulations and experimental data. The discrepancy is manifested as some reduction and broadening, as well as a slight deformation of the measured intensity peaks compared to the simulated ones. For instance, for the case of 1-spot, the theoretical intensity maximum is 106.5 and the spot has a round shape with the half-maximum diameter of $1.4 \mathrm{~mm}$. In the experiment, the peak magnitude decreases down to 76.5 and the spot appears to be elongated along the $\mathrm{Y}^{\prime}$ axis having a quasielliptical shape with half-maximum dimensions of $1.9 \times 2.5 \mathrm{~mm}$. For the case of 4-spots focusing, the GSA computations converged to focal intensity profiles with unequal peak magnitudes distributed as $22.3 \& 27.2$ for the peaks located on the horizontal axis of the cross, and $24.6 \& 23.8$ for the peaks on the vertical axis. All simulated peaks are round-shaped with half-maximum diameter of $1.2 \mathrm{~mm}$. It is noteworthy that the measurements of the 4-spots case showed qualitatively similar non-uniform peak distribution, also confirming the $\mathrm{Y}^{\prime}$-elongation phenomenon that also appears in the 1-spot case. The experimental magnitudes and dimensions of the 4-spots are shown in Table 1.

\section{Discussion of the results}

Despite a possible distortion of the wave field intensity registered by a pyro-detector, the main reason to explain the effects of the magnitude reduction, spatial broadening and deformation of the focal peaks compared to the theoretical predictions is presumably related to phase errors. These arise inevitably when the correspondence between the phase and the unit cell geometry is established through the modeling of each metasurface as a spatially-uniform $2 \mathrm{D}$ array (see Fig. 3). However, in the practical realization each unit cell operates in nonuniform environment due to the gradual geometrical variation of the neighboring cells. This yields deviations from the theoretical phase values presented in Fig. 3. Such deviations can be noticeable in the pixels where the reflection phase $\varphi_{H R A}(x, y)$ switches 
(a)
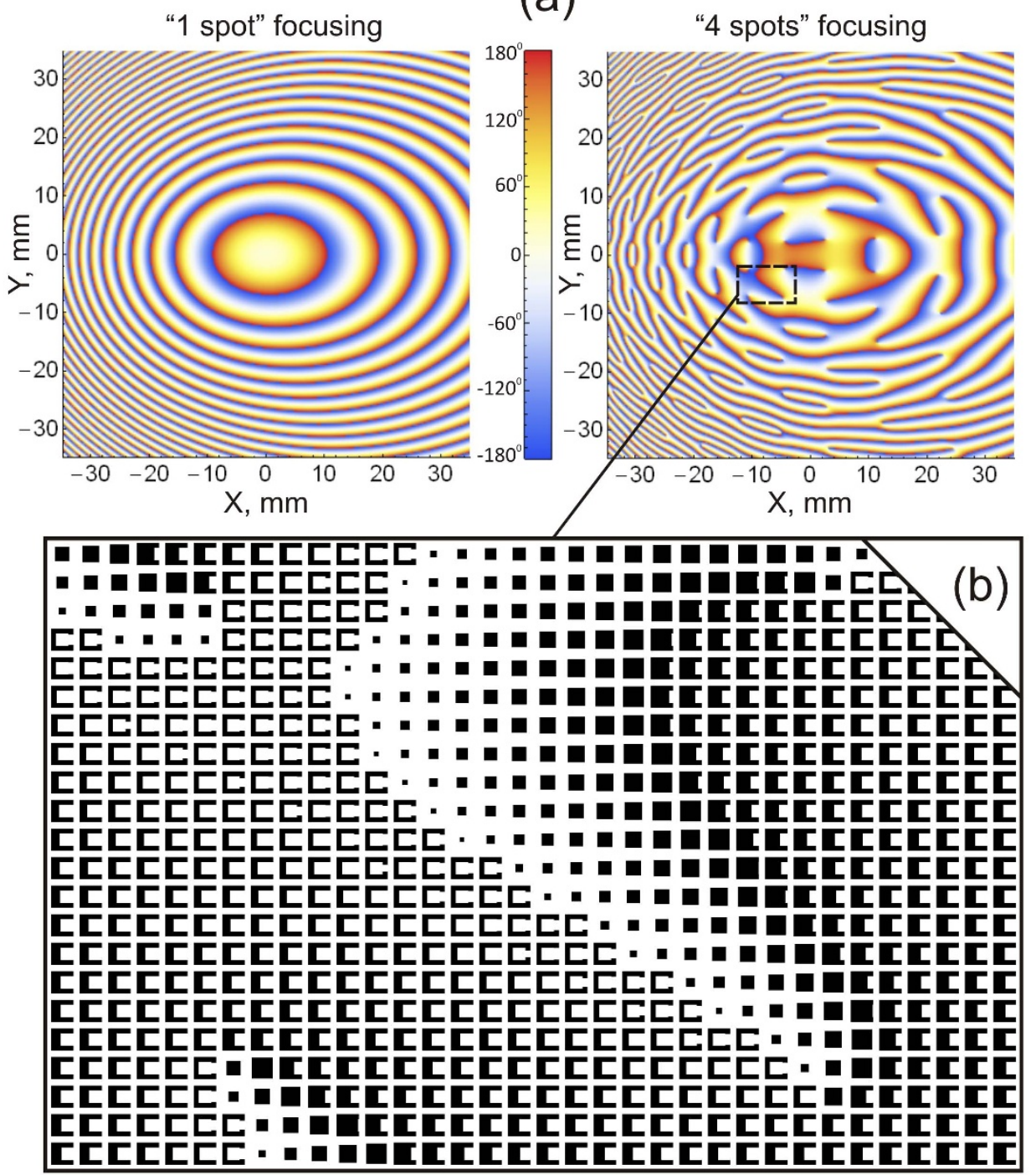

Figure $4 \mid$ Metasurface patterns. (a) Distributions of the reflection phase over HRA surfaces for the cases of 90 deg reflective focusing into a single spot (left) and four spaced spots (right) computed for the focal distance of $F=60 \mathrm{~mm}$ and the operating frequency of $0.35 \mathrm{THz}$. (b) Fragment of the metasurface pattern for 4-spots HRA adapted to TE-polarized excitation. Metallized areas are shown by black.

from $180 \mathrm{deg}$ to $-180 \mathrm{deg}$, i.e. where the topological pattern drastically changes: from patches of the first topological numbers to SRRs of the last ones (see "white-looking" areas faintly-filled with metal in Fig.4(b)). A correct evaluation of the mentioned phase errors lies beyond the scope of this paper; our goal herein is to demonstrate that, despite phase errors high-performance terahertz focusing devices, as proven by Fig. 6(b).

For practical applications, three additional important parameters of the focusing devices are usually analyzed: diffraction efficiency, spectral bandwidth, and, in case of utilizing polarization-dependent metasurfaces, sensitivity of focusing to variation of the incident polarization. In general, the DOE's diffraction efficiency (DE) is defined as the ratio of the power diffracted into a chosen diffraction order to the power of the incoming wave beam. For the case of 1-spot, the direct integration of the focal plane intensity distribution $I_{F P}$ $\left(x^{\prime}, y^{\prime}\right)$ over the round area centered in the focal peak and diameter equal to three half-maximum diameters results in a DE equal to $93 \%$ and $80 \%$ for the theoretical and experimental cases, respectively. Similarly, the DE of 4 -spots is $86 \%$ and $78 \%$ for the theoretical and experimental cases, respectively. These relatively high $\mathrm{DE}$ values are achieved thanks to using subwavelength pixels $\left(\lambda_{0} / g \cong 3\right)$ that minimize the staircase variation step for the reflection phase $\varphi_{H R A}(x, y)$ and lead to diminishing the spurious diffraction losses. This fact, makes the designed metasurfaces very attractive and promising for practical applications. Diffraction losses could be further reduced by increasing the $\lambda_{0} / g$ ratio so that the focal intensity profile is improved.

Regarding sensitivity of focusing to the incident polarization, both designs exhibit quite robust behavior: the magnitude of the foci decreases to half the maximum only when the exciting polarization changes at \pm 40 deg relative to the optimal (TE) state. Additionally, our investigations show that, when properly designed for TMpolarized excitation, the metasurfaces keep all the main focusing characteristics obtained for TE-polarization, excluding the additional power losses, which are estimated on the level of $3-5 \%$ and are attributed to cross-polarization scattering.

The spectral properties of the metasurfaces and, in particular, their operational frequency bandwidth (BW) are illustrated in Fig. 7 where the frequency dependences of the diffraction efficiency and the normalized focal peak magnitudes (NFPM) are shown. Referred to $-3 \mathrm{~dB}$ level, DE-BW is evaluated as the frequency range where DE values are above half of their maxima. Relative to the frequency of $0.35 \mathrm{THz}$, both theoretical and experimentally measured DE-BW are estimated around $19 \%$ for 1 -spot and $15 \%$ for 4 -spots. Unlike DE, which is the area-integrated characteristics, NFPM is more sensitive 


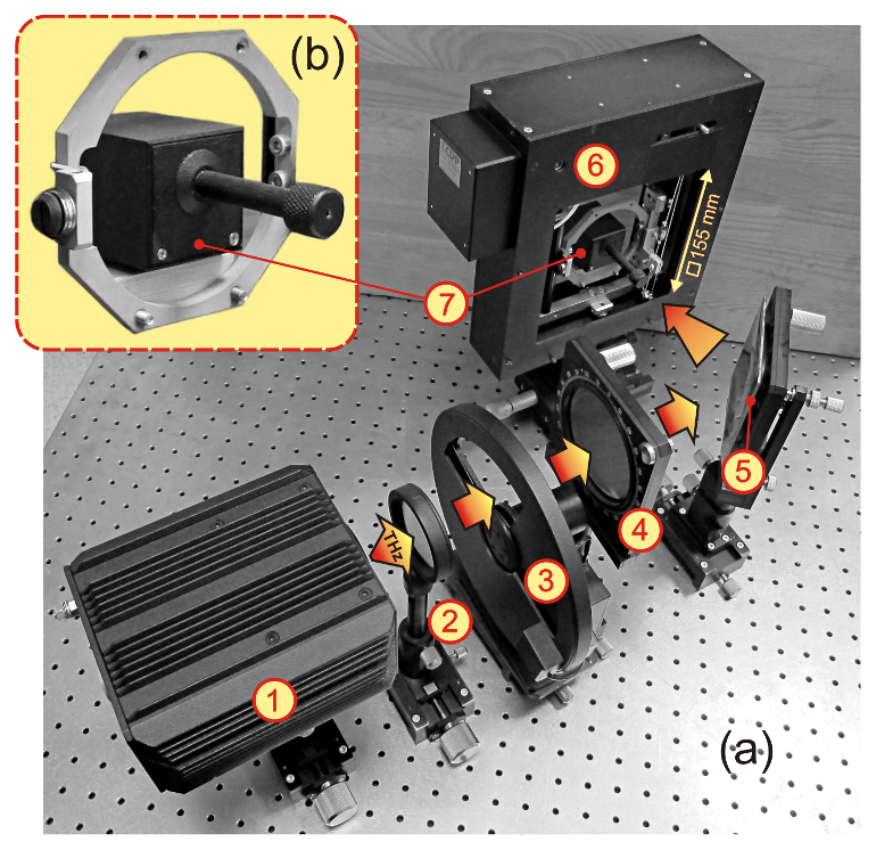

Figure $5 \mid$ Quasi-optical experimental setup. (a) Photograph of the experimental setup used for measuring the focusing performance of the developed HRAs: (1) - THz BWO, (2) - beam forming lens, (3) - chopper, (4) - wire grid polarizer, (5) - HRA (fixed to the optical holder), (6) - 2D raster scanner, (7) - pyroelectric detector. (b) Magnified photo of the pyro-detector mounted on the carrying metallic frame.

to a frequency variation. Moreover, in the case of 4-spots each one of the peaks degrades slightly differently, resulting in some variation of the individual NFPM-BW (see Fig. 7 (right)). Referred to $-3 \mathrm{~dB}$ decay relative to the peak magnitude, the theory and experiment yield the concordant values of NFPM-BW: $14 \%$ for 1-spot and on average $9.4 \%$ for 4 -spots (it actually varies from $8.8 \%$ to $10 \%$ for different peaks).

Similar to reflectarrays ${ }^{4,14,15}$, the spectral performance of the holographic metasurfaces is limited by two factors: the bandwidth of the metasurface unit cells, and a differential spatial phase delay resulting from different path lengths from the focal spots to each point of the metasurface. To gain a deeper insight on the phase mismatching, we may consider two arbitrary metasurface points $A$ and $B$ having the reflection phases $\varphi_{0}^{A}$ and $\varphi_{0}^{B}$ respectively, which are specified for the optimized frequency $v_{0}=0.35 \mathrm{THz}$. Limiting our consideration to 1-spot focusing, the phase relation for ideal focusing can be formulated as $\left(\varphi_{0}^{B}-\varphi_{0}^{A}\right)-2 \pi v_{0}\left(r^{B}-r^{A}\right) / c=2 m \pi$, where $r^{A}$ and $r^{B}$ are the distances between the focal point and the points $A$ and $B$ respectively, $c$ is the speed of light in vacuum, and $m$ is an integer. To make the focal plane intensity independent on frequency (implying a constant incident beam profile, for simplicity), we should impose the requirement to fulfill this relation at any other frequency $v$ where the $A$ and $B$ reflection phases are equal to $\varphi^{A}$ and $\varphi^{B}$ respectively: $\left(\varphi^{B}-\varphi^{A}\right)-2 \pi \nu\left(r^{B}-\right.$ $\left.r^{A}\right) / c=2 m \pi$. These two relations can be reduced upon elimination of $\boldsymbol{r}^{A}$ and $\boldsymbol{r}^{B}$ to the next equation:

$$
\Delta \varphi^{B A}=\Delta \varphi_{0}^{B A} \cdot v / v_{0}+2 m \pi \cdot\left(\mathbf{1}-v / v_{0}\right),
$$

where $\Delta \varphi^{B A}=\left(\varphi^{B}-\varphi^{A}\right), \Delta \varphi_{0}^{B A}=\left(\varphi_{0}^{B}-\varphi_{0}^{A}\right)$.

The first term on the right side of (1) describes the ideal frequency dispersion law for the metasurface unit cells necessary to compensate a phase shift between surface points when changing the frequency. However, such compensation will work for the whole surface only if all the partial waves arriving at the focal point from the metasurface have the relative phase shift modulus not exceeding $360^{\circ}$. This corresponds to the condition $m=0$ and can be realized when the metasurface transverse size $D$ is relatively small compared to the focal

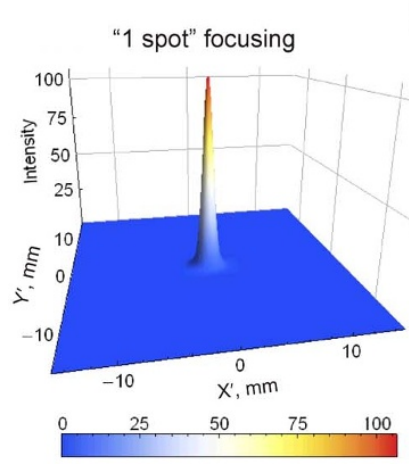

(a)
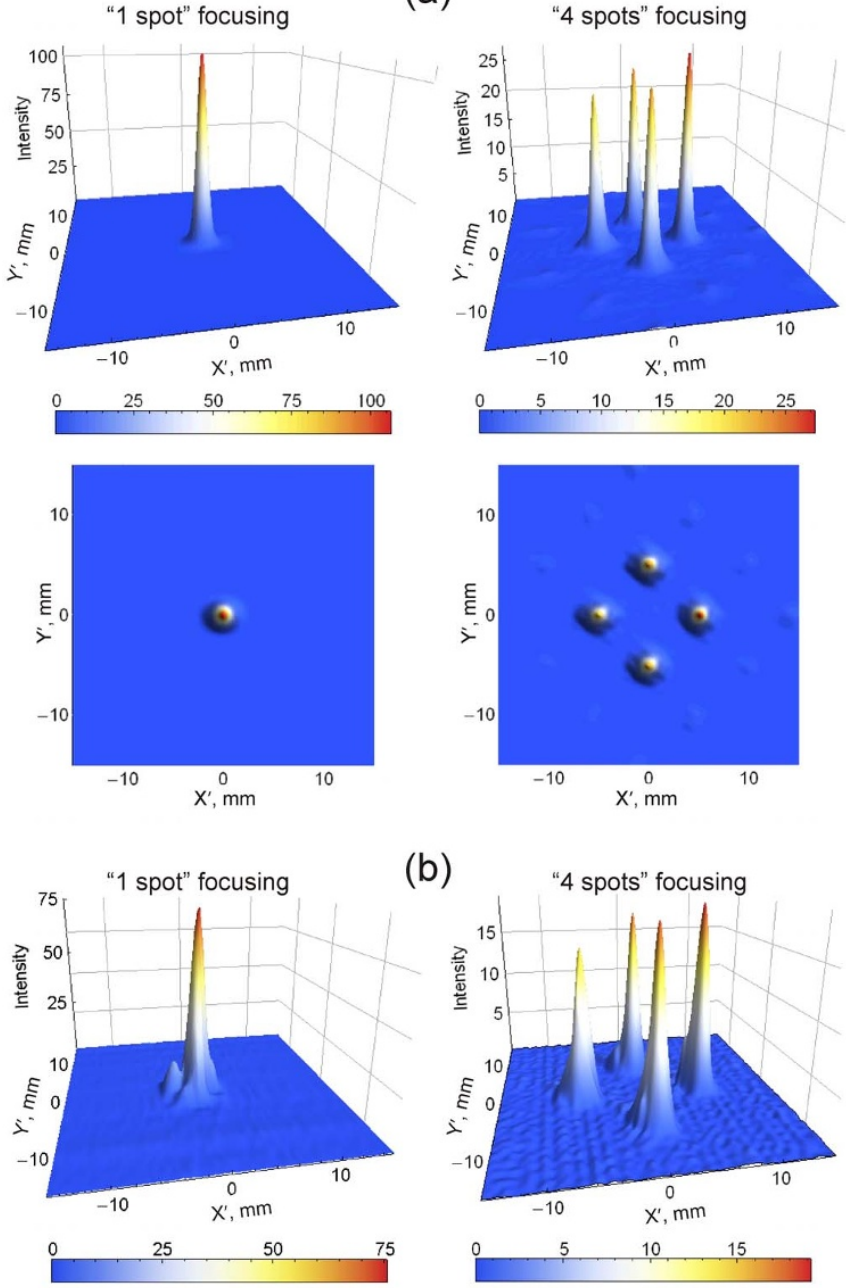

(b)
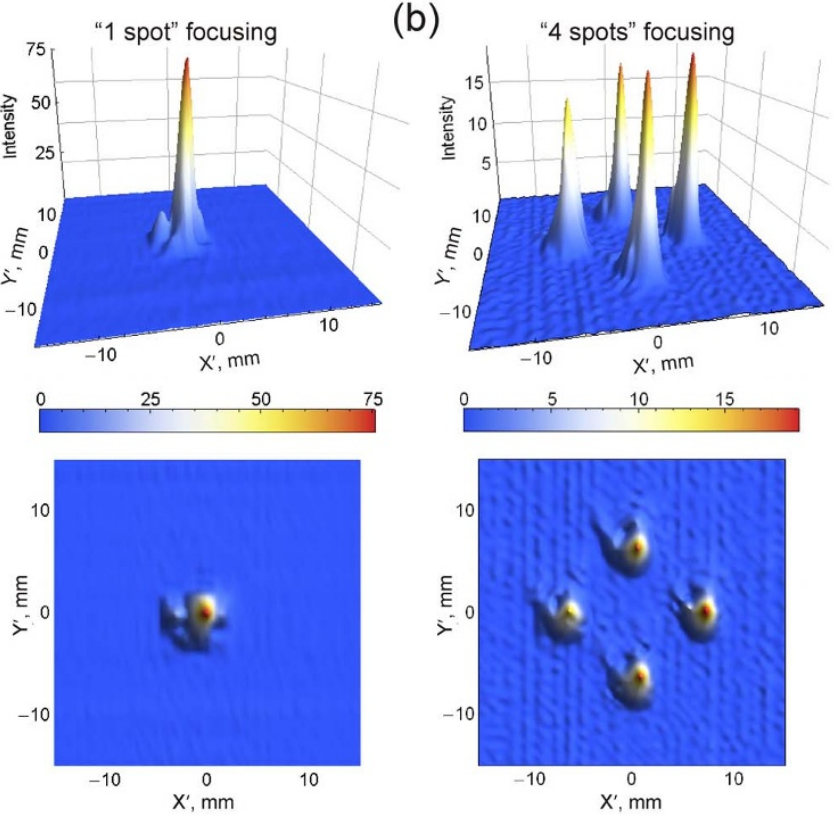

Figure 6 | Focusing performance of the holographic metasurface.

Theoretical (a) and experimentally measured (b) intensity distributions in the focal plane $\left(\mathrm{X}^{\prime}, \mathrm{Y}^{\prime}\right)$ obtained for $90^{\circ}$ reflective focusing into a single spot (left) and four spots (right) at the frequency of $0.35 \mathrm{THz}$.

distance $F\left(D<\left(8 \lambda_{0} F\right)^{1 / 2}\right)$. For the case $D / F \cong 1$, the second term on the right side of (1) becomes non-zero and cannot be neglected. It introduces a supplementary non-compensable phase shift which increases when $|m|$ grows and results in an extra narrowing of the

Table 1 | Characteristics of the spots in the 4-spots experiment

\begin{tabular}{|lcccc|} 
& \multicolumn{4}{c}{ Peak } \\
\cline { 2 - 5 } & Left & Right & Bottom & \multicolumn{1}{c}{ Top } \\
\hline Normalized intensity & 15.3 & 19.2 & 18.4 & 17.9 \\
Half-maximum & $2.5 \times 3.4$ & $2.7 \times 3.5$ & $2.3 \times 3.4$ & $2.5 \times 3.4$ \\
dimensions $(\mathrm{mm})$ & & & & \\
\hline
\end{tabular}



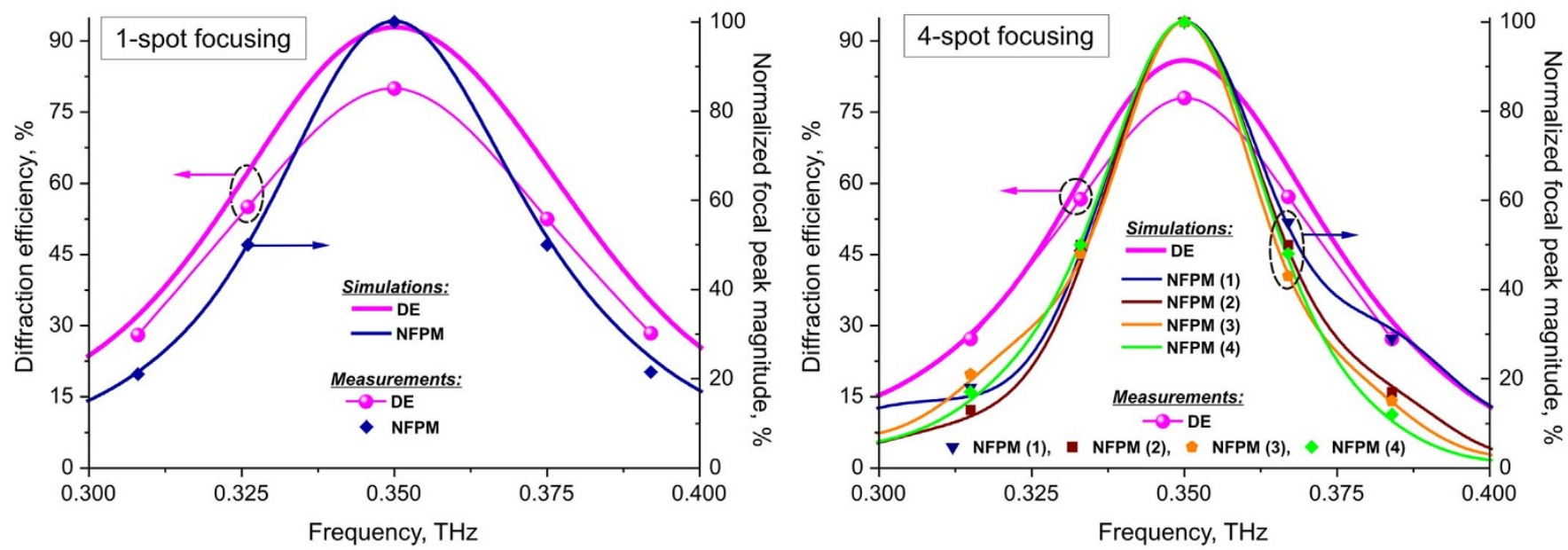

Figure 7 Focusing performance of the holographic metasurfaces as a function of frequency. Spectral behavior of the diffraction efficiency and focal peak magnitude normalized to its maximum when focusing into a single spot (left) and four spots (right). For 4-spots, the peaks from Fig. 6 (right) are numerated as follows: 1 - left, 2 - right, 3 - bottom, 4 - top.

bandwidth for the metasurfaces having a large number of the operating zones shifted in phase by $360^{\circ}$ (see Fig. 4(a)). In this work, at least four of such zones should be taken into account as the metasurfaces are illuminated by the wave beam with the half-maximum dia- meter $w \cong 18 \mathrm{~mm}$. To quantify both terms in equation (1) applied to the "Patch-to-SRR" morphing from Fig. 3 and, thus, to get information on the phase mismatch corresponding to the actual values of the metasurface bandwidth, we will consider the quantities $\Delta \varphi^{B A}$ and $\Delta \varphi_{0}^{B A}$ as
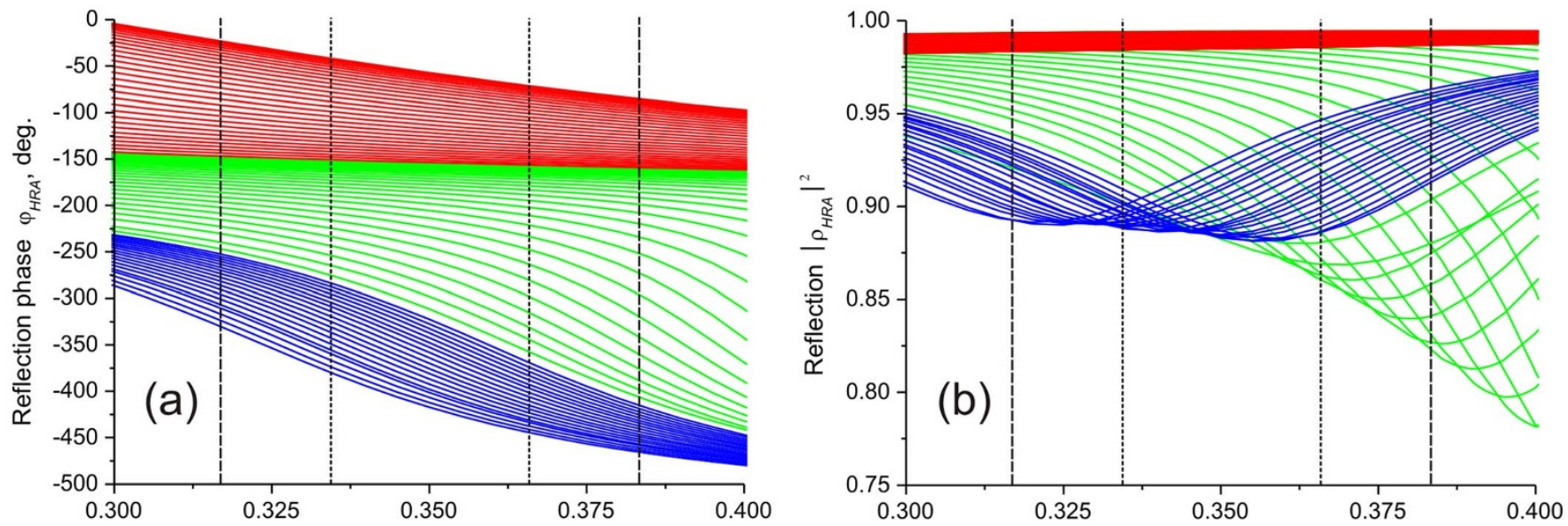

Frequency, THz - Patches - USRs - SRRs

Frequency, $\mathrm{THz}$
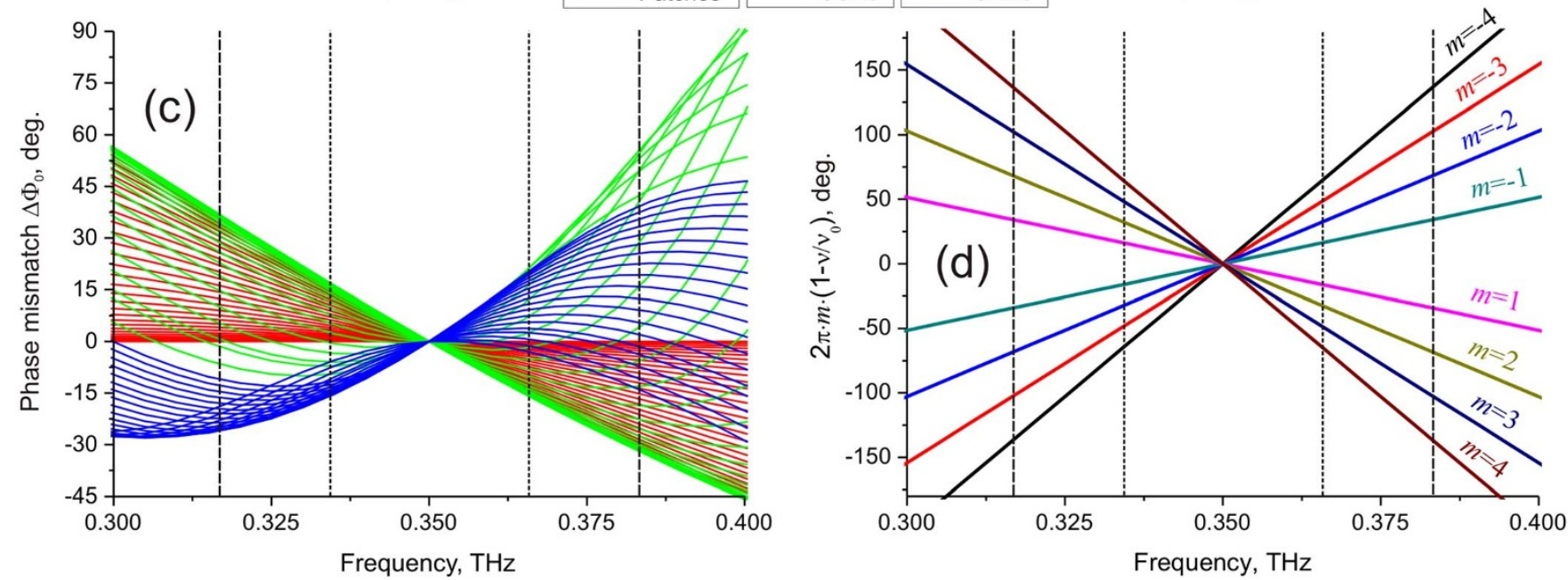

Figure 8 Spectral properties of the uniform-metasurface-based reflectarrays. (a) The reflection phase; (b) the reflection coefficient; (c) phase mismatch function $\Delta \Phi_{m}$ for $m=0$, and (d) extra factor $2 m \pi \cdot\left(1-v / v_{0}\right)$ for different $m$. TE polarization, $45^{\circ}$ oblique incidence. The vertical lines delimit $19 \%$ (dash) and $9 \%$ (short dash) bandwidths. 
Table 2 | Calculated boundary and mean values for the phase mismatch function $\Delta \Phi_{0}$

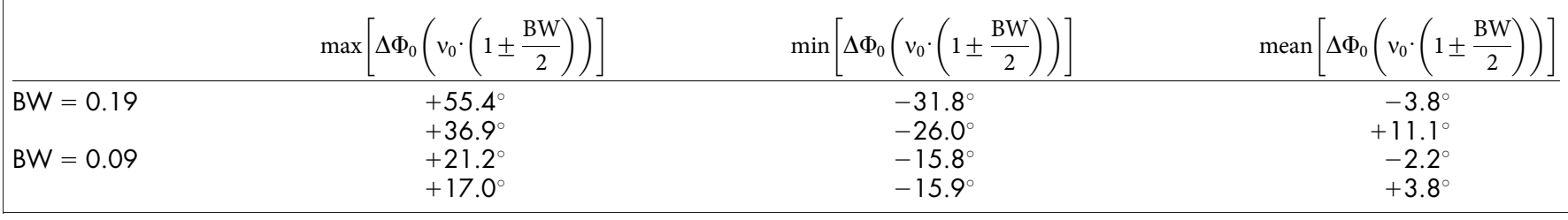

the differences between the TE reflection phases of the 1st number topology (the patch of the smallest size) and the variable topological number $X(X \in[1,78])$ determined at the specified frequencies: $\Delta \varphi^{B A} \equiv \Delta \varphi^{\text {IvSX }}(v)=\varphi^{\text {Topoll }}(v)-\varphi^{\text {TopolX }}(v), \quad \Delta \varphi_{0}^{B A} \equiv \Delta \varphi^{\text {IvSX }}\left(v_{0}\right)=\varphi^{\text {Topoll }}\left(v_{0}\right)-$ $\varphi^{\text {TopolX }}\left(v_{0}\right)$. Figures $8(a)$, (b) illustrate the frequency dispersion of the complex TE-reflectance for the full morphology set comprising 78 topological variants of the uniform-metasurface-based reflectarrays from Fig. 3 computed in the range of $0.3-0.4 \mathrm{THz}$. Introducing the phase mismatch function $\Delta \varphi_{m}$

$\Delta \Phi_{m}(v)=\Delta \varphi^{1 v s X}(v)-\left[\Delta \varphi^{1 v s X}\left(v_{0}\right) \cdot v / v_{0}+2 m \pi \cdot\left(1-v / v_{0}\right)\right]$,

to describe a deviation of the actual phase shift $\Delta \varphi^{1 v s x}(v)$ between the two metasurface points having the local topological numbers 1 and $X$ from the ideal phase shift specified by (1), we can further track out the spectral behavior of $\Delta \Phi_{\mathrm{m}}$ using the dispersion curves from Fig. 8(a). Fig. 8(c) demonstrates the numerical results for the case of $m=0$, while Fig $8(\mathrm{~d})$ represents the extra term $2 m \pi \cdot\left(1-v / v_{0}\right)$ for $m$ $\neq 0$. One can see a relatively rapid variation of the functions $\Delta \Phi_{0}$ versus frequency which, in addition, behave differently for different topologies. The spread of $\Delta \Phi_{0}$ magnitudes is summarized in Table 2 and provides information on the minimal, maximal, and mean values (uniformly averaged over all 78 topologies) referred to the boundary frequencies of the broadest and narrowest bands determined in this work: $19 \%$ (1-spot DE-BW) and 9\% (4-spots NFPMBW) bandwidths respectively. We deduce from Table 2 that a $\Delta \Phi_{0}$ magnitude up to $\sim 30-50^{\circ}$ is allowable to realize DE with $19 \%$ bandwidth, while only $\sim 15-20^{\circ}$ are permissible for $9 \%$ of NFPMBW. Note that such values of the phase mismatch are achieved when several zones with different $m$ jointly work in our metasurfaces. For each zone with $m \neq 0$, the phase mismatch is increased by an extra factor $\mp 180^{\circ} \cdot \mathrm{m} \cdot \mathrm{BW}$. The values of this extra factor for different $m$ are summarized in Table 3. By decreasing the number of actually operating zones through reducing the $D / F$ ratio, the bandwidth can be expanded.

To conclude, in this work a new type of planar holographic metasurfaces (or reflectarrays) designed for simple and sophisticated focusing of THz radiation using "Patch-to-SRR" topological morphing of the metasurface pattern is proposed and experimentally investigated at the frequency of $0.35 \mathrm{THz}$. A TE-polarization scheme is chosen judiciously to reduce the cross-polarization losses that are susceptible to arise in anisotropic unit cells such as the USRs and SRRs. The experimental measurement confirms the theoretical predictions with a good degree of concordance and shows that the holographic metasurfaces enable to realize the operation bandwidth up to $20 \%$ in the non-paraxial configuration with $F / D \cong 1$. The considered approach demonstrates the high-performance solution

Table 3 | Calculated extra factor $2 m \pi \cdot\left(1-v / v_{0}\right)$ for different $m$

\begin{tabular}{lllll} 
& $m= \pm 1$ & $m= \pm 2$ & $m= \pm 3$ & $m= \pm 4$ \\
\hline $\mathrm{BW}=0.19$ & $\mp 34.2^{\circ}$ & $\mp 68.4^{\circ}$ & $\mp 102.6^{\circ}$ & $\mp 136.8^{\circ}$ \\
$\mathrm{BW}=0.09$ & $\mp 16.2^{\circ}$ & $\mp 32.4^{\circ}$ & $\mp 48.6^{\circ}$ & $\mp 64.8^{\circ}$
\end{tabular}

with the experimentally measured diffraction efficiency around $80 \%$ for creating purely flat, thin, light-weight and relatively inexpensive passive beam-shaping and beam-focusing devices for the THz band.

\section{Methods}

Gerchberg-Saxton iterative algorithm. For reflecting DOEs, here generically referred to as holographic reflectarrays (HRAs), GSA ${ }^{19-21}$ starts with computing the electric field amplitude distribution $E_{0}(x, y)$ for the wavefront in the HRA plane (X, Y) using the beam intensity distribution $I_{0}\left(x_{0}, y_{0}\right)$ given in the beam-waist plane $\left(\mathrm{X}_{0}, \mathrm{Y}_{0}\right)$ (see Fig. 1,2). The field amplitude $E_{0}(x, y)$ is related to the wave intensity $I_{0}(x, y)$ and phase $\phi_{0}(x, y)$ as $E_{0}(x, y)=\left(8 \pi I_{0}(x, y) / c\right)^{1 / 2} \cdot \exp \left(\mathrm{j} \phi_{0}(x, y)\right)$, where $c$ is the speed of light. For a Gaussian beam, the transition from $I_{0}\left(x_{0}, y_{0}\right)$ to $I_{0}(x, y)$ and $\phi_{0}(x, y)$ is readily realized with the help of classical Gaussian optics formulas ${ }^{6}$. Knowing $E_{0}(x, y)$, the field reflected from the HRA at every surface point $(x, y)$ is computed afterwards via multiplying $E_{0}(x, y)$ by the HRA's complex reflectance $\rho_{H R A}^{(m)}(x, y) \cdot \exp \left(\mathrm{j} \varphi_{H R A}^{(m)}(x, y)\right)$, where $\rho_{H R A}(x, y)$ and $\varphi_{H R A}(x, y)$ correspond to the reflection amplitude and phase respectively, while the superscript $(m)$ indicates the iteration number. It is assumed that $\rho_{H R A}(x, y)$ is a known function of $\varphi_{H R A}(x, y)$, thus only the unknown distribution $\varphi_{\text {HRA }}(x, y)=\lim _{m \rightarrow \infty} \varphi_{H R A}^{(m)}(x, y)$ is to be determined via a GSA iterative procedure. For the first iteration $(m=1)$, the distribution $\varphi_{H R A}^{(m)}(x, y)$ is chosen to be arbitrary (e.g. random).

As soon as the distribution of the wave field reflected from the HRA is established for the iteration $m$, it is further used to figure out the wave field in the focal plane $\left(\mathrm{X}^{\prime}\right.$, $\mathrm{Y}^{\prime}$ ) by applying a "forward propagator" operator, which is basically represented by the Fresnel-Kirchhoff integral ${ }^{31}$. The computed focal plane intensity distribution $I_{F P}^{(m)}$ $\left(x^{\prime}, y^{\prime}\right)$ is compared afterwards with the desired intensity distribution $I_{F P}\left(x^{\prime}, y^{\prime}\right)$. If the maximal relative error $\max \left(\left|I_{F P}^{(m)}\left(x^{\prime}, y^{\prime}\right)-I_{F P}\left(x^{\prime}, y^{\prime}\right)\right| / I_{F P}\left(x^{\prime}, y^{\prime}\right)\right)$ over the focal area exceeds the prescribed convergence limit or the maximally acceptable number of iterations is not achieved, the iterative process continues to the stage $(m+1)$. From this point, the computed focal plane field $\left(8 \pi I_{F P}^{(m)}\left(x^{\prime}, y^{\prime}\right) / c\right)^{1 / 2} \cdot \exp \left(\mathrm{j} \varphi_{F P}^{(m)}\left(x^{\prime}, y^{\prime}\right)\right)$ is modified by substituting $I_{F P}^{(m)}\left(x^{\prime}, y^{\prime}\right)$ for $I_{F P}\left(x^{\prime}, y^{\prime}\right)$ and is further sent back to HRA. By applying the Fresnel-Kirchhoff integral to such backward propagation of the wavefront from the focal plane to HRA, one can calculate the modified field distribution in the HRA plane $(\mathrm{X}, \mathrm{Y})$ and therefore the modified HRA reflection phase $\varphi_{H R A}^{(m+1)}(x, y)$. The latter one is derived from the resulting surface phase distribution after subtracting the $\phi_{0}(x, y)$ factor originated from the incident Gaussian beam. The iterative loop closes when the distribution $\varphi_{H R A}^{(m)}(x, y)$ is replaced by $\varphi_{H R A}^{(m+1)}(x, y)$ and the iterative procedure continues. The iterative process repeats until the computed focal plane intensity distribution maximally conforms to the desired distribution $I_{F P}$ $\left(x^{\prime}, y^{\prime}\right)$. In this case, the incident beam intensity distribution retrieved on the "backward" branch of the iterative loop should fit the original distribution $I_{0}\left(x_{0}, y_{0}\right)$, and the resulting phase function $\varphi_{H R A}(x, y)$, i.e. the required "computer-generated hologram", is obtained.

Full-wave simulation. As the direct full-wave modeling of oversized HRA composed of non-uniform unit cells requires enormous amount of computational resources, a good approximation is to assume local periodicity. That is, to reduce the problem to modeling uniform RAs differed in metasurface patterns and then to synthesize the output HRA using the correspondence between the RA's reflection phase and its metasurface unit cell geometry. In this work, 78 variants of regular RAs with different metasurface patterns, covering the full transformation cycle from Fig. 3(a), were numerically optimized in ASYS HFSS ${ }^{\mathrm{TM}}$ electromagnetic software to achieve the optimal performance for the output HRA excited by the electromagnetic wave polarized transversely to USR and SRR gaps. All RAs were modeled as infinite regular 2D arrays with identical $x$ and $y$ lattice constants $g$ by exploiting the regime of Floquet ports and periodic boundary conditions applied to the RA unit cell. Due to fabrication limitations, the restriction of $56 \mu \mathrm{m}$ for the minimal width of the metallized strips, as well as the inter-element spaces in metasurface patterns was imposed in the geometry optimization. As a result, for the PP slab $190 \mu \mathrm{m}$ thick, the minimized unit cell dimensions $g \times g$ providing $360 \mathrm{deg}$ of the reflection phase variation $\Delta \varphi_{\mathrm{RA}}$ at the operation frequency of $0.35 \mathrm{THz}$ and TE-polarized excitation were found to be $286 \times$ $286 \mu \mathrm{m}$. Thus, the subwalengthness factor, defined as the ratio of the free space wavelength $\lambda_{0}$ to the unit cell periodicity $g$, is evaluated as $\lambda_{0} / g \cong 3$. 
Experimental setup. The experimental scheme for terahertz focusing by the HRA investigated in this work is shown in Figs. 1 and 5. The metasurface is lithographically patterned on a grounded dielectric slab and is illuminated by a Gaussian beam from a backward-wave oscillator (BWO) under an incidence angle of $45 \mathrm{deg}$. After reflection from the metasurface and a proper amplitude-phase transformation, the wavefront is focused afterwards on the focal plane, which was chosen to be positioned at the distance of $F=60 \mathrm{~mm}$ from the metasurface center, while its normal is oriented at 90 deg relative to the propagation direction of the incident beam ("90 deg-reflection scheme"). In our experiments, the metasurface is square with overall dimensions of $D$ $\times D=70 \times 70 \mathrm{~mm}^{2}$, which are almost fourfold larger than the incident beam diameter $w$ evaluated as $18 \mathrm{~mm}$ at half maximum of the beam intensity.

The measurements (see Fig. 6) were done with a pyroelectric detector mounted on the tip of a metallic rod with the outer diameter of $15 \mathrm{~mm}$ installed on a computercontrolled $2 \mathrm{D}$ raster scanner. The step of $2 \mathrm{D}$ raster scanning along both axes was $0.43 \mu \mathrm{m}$ (i.e., $0.5 \lambda_{0}$ ). The pyro-detector developed by CDP System Corp ${ }^{32}$ was implemented to have the receiving window diameter of $1.5 \mathrm{~mm}$.

1. Yun-Shik, L. Principles of Terahertz Science and Technology (Springer, New York, 2009).

2. Holloway, C. L. et al. An Overview of the Theory and Applications of Metasurfaces: The Two-Dimensional Equivalents of Metamaterials. IEEE Antennas Propag. Mag. 54, 10-35 (2012).

3. Munk, B. A. Frequency Selective Surfaces: Theory and Design (John Wiley \& Sons, New York, 2000)

4. Balanis, C. A. Modern Antenna Handbook, Hoboken (John Wiley \& Sons, NJ, 2008).

5. Pfeiffer, C. \& Grbic, A. Metamaterial Huygens' Surfaces: Tailoring Wave Fronts with Reflectionless Sheets. Appl. Phys. Lett. 110, 197401-1-5 (2013).

6. Goldsmith, P. F. Quasioptical Systems: Gaussian Beam Quasioptical Propagation and Applications (IEEE Press, Piscataway, NJ, 1998).

7. Aznabet, M. et al. Polypropylene-substrate-based SRR-and CSRR-metasurfaces for submillimeter waves. Opt. Express 16, 18312-18319 (2008).

8. $\mathrm{Hu}, \mathrm{D}$. et al. Ultrathin Terahertz Planar Elements. Adv. Opt. Mat. 1, 186-191 (2013).

9. Aieta, F. et al. Aberration-Free Ultrathin Flat Lenses and Axicons at Telecom Wavelengths Based on Plasmonic Metasurfaces. Nano Lett. 12, 4932-4936 (2012).

10. Ni, X., Kildishev, A. V. \& Shalaev, V. M. Metasurface holograms for visible light. Nature Comm. 4, 2807 (2013).

11. Huang, L. et al. Three-dimensional optical holography using a plasmonic metasurface. Nature Comm. 4, 2808 (2013)

12. Salem, M. A. \& Caloz, C. Manipulating light at distance by a metasurface using momentum transformation. Opt. Express 22, 14530-14543 (2014).

13. Zhao, J., Li, B., Chen, Z. \& Qiu, C.-W. Manipulating Acoustic Wavefront by Inhomogeneous Impedance and Steerable Extraordinary Reflection. Sci. Rep. 3, 2537 (2013).

14. Huang, J. \& Encinar, J. A. Reflectarray Antennas (John Wiley \& Sons, Hoboken, NJ, 2008).

15. Shaker, J., Reza Chaharmir, M. \& Ethier, J. Reflectarray Antennas: Analysis, Design, Fabrication, and Measurement (Artech House, Boston, 2013).

16. Vinokurov, N. A. et al. Diffractive optical elements and quasioptical schemes for experiments on a high-power terahertz free-electron laser. Radiophys. Quant. Electron. 50, 803-812 (2007).

17. Vedernikov, V. M. et al. Diffractive Elements for a Free Electron Laser. Optoelectron. Instrum. Data Proc. 46, 365-375 (2010).

18. Agafonov, A. N. et al. Silicon diffractive optical elements for high-power monochromatic terahertz radiation. Optoelectron. Instrum. Data Proc. 49 189-195 (2013).

19. Gerchberg, R. W. \& Saxton, W. O. A practical algorithm for the determination of the phase from image and diffraction plane pictures. Optik 35, 237-242 (1972).

20. Soifer, V., Kotlyar, V. \& Doskolovich, L. Iterative methods for diffractive optical elements computation (Taylor \& Francis, London, 1997).

21. Soifer, V. A. Methods for Computer Design of Diffractive Optical Elements (John Wiley \& Sons, New York, 2002).
22. Pozar, D. M. Wideband reflectarrays using artificial impedance surfaces. Electron Lett. 43, 148-149 (2007).

23. Luo, L. et al. Broadband terahertz generation from metamaterials. Nature Comm. 5, 1-6 (2014)

24. Marqués, R., Martín, F. \& Sorolla, M. Metamaterials with Negative Parameters: Theory, Design, and Microwave Applications (Wiley, Hoboken, NJ, 2008).

25. Padilla, W. J., Taylor, A. J., Highstrete, C., Lee, M. \& Averitt, R. D. Dynamical Electric and Magnetic Metamaterial Response at Terahertz Frequencies. Phys. Rev. Lett. 96, 107401-1-4 (2006).

26. Wan, X., Jiang, W. X., Ma, H. F. \& Jun Cui, T. A broadband transformation-optics metasurface lens. Appl. Phys. Lett. 104, 151601-1-4 (2014).

27. Yang, Y., Mandehgar, M. \& Grischkowsky, D. Broadband THz Pulse Transmission Through the Atmosphere. IEEE T. THz Sci, Tech. 1, 264-273 (2011).

28. Kuznetsov, S. A. et al. Regular and Anomalous Extraordinary Optica Transmission at the THz-gap. Opt. Express 17, 11730-11738 (2009).

29. Navarro-Cía, M. et al. Route for bulk millimeter wave and terahertz metamaterial design. IEEE J. Quant. Electron. 47, 375-385 (2011).

30. Beruete, M., Sorolla, M., Marqués, R., Baena, J. D. \& Freire, M. J. Resonance and cross-polarization effects in conventional and complementary split ring resonator periodic screens. Electromagnetics 26, 247-260 (2006).

31. Born, M. \& Wolf, E. Principles of Optics (Cambridge University Press, Cambridge, UK, 1999).

32. CDP System Corporation, http://www.teraspectr.ru/ Date of access: 24/11/2014.

33. Kozlov, G. V. \& Volkov, V. V. [Coherent Source Submillimeter Wave Spectroscopy] Millimeter and Submillimeter Wave Spectroscopy of Solids [Grüner, G. (ed.)] [51-109] (Springer-Verlag, Berlin, Heidelberg, 1998).

\section{Acknowledgments}

In memoriam Prof. Mario Sorolla. This work was supported by the Ministry of Education and Science of the Russian Federation under the State Assignment Contract \#3002 (implementation of the Gerchberg-Saxton iterative algorithm and experimental testing), the Russian Science Foundation under the Project 14-12-01037 (full-wave electromagnetic simulations), and the Spanish Government under contracts Consolider "Engineering Metamaterials" CSD2008-00066, and TEC2011-28664-C01. M. Beruete acknowledges funding by the Spanish Government under the research contract program Ramón y Cajal RYC-2011-08221. M. N.-C. was supported by Imperial College London through a Junior Research Fellowship.

\section{Author contributions}

S.K. conceived the idea and supervised the study. M.A.A. implemented the Gerchberg-Saxton iterative algorithm. S.K., M.A.A. and M.N.-C. contributed to the numerical results. S.K. fabricated the samples. S.K. and M.A.A. performed the experiment and data analysis. S.K., M.A.A., M.B. and M.N.-C. contributed to the discussion. S.K., M.A.A., M.B. and M.N.-C. wrote the paper.

\section{Additional information}

Competing financial interests: The authors declare no competing financial interests. How to cite this article: Kuznetsov, S.A., Astafev, M.A., Beruete, M. \& Navarro-Cía, M. Planar Holographic Metasurfaces for Terahertz Focusing. Sci. Rep. 5, 7738; DOI:10.1038 srep07738 (2015)

This work is licensed under a Creative Commons Attribution-NonCommercialShareAlike 4.0 International License. The images or other third party material in this article are included in the article's Creative Commons license, unless indicated otherwise in the credit line; if the material is not included under the Creative Commons license, users will need to obtain permission from the license holder in order to reproduce the material. To view a copy of this license, visit http:// creativecommons.org/licenses/by-nc-sa/4.0/ 